\title{
Targeted agents in gynecologic cancers
}

\section{Editorial}

In 21 st century, cancer has become one of the leading causes of death especially in developed countries. On the other hand, gynecologic malignancies take an important part in women cancers that uterine corpus and ovarian cancers are in the ten leading cancer types for both new cases and deaths. While endometrial cancer is the most common gynecologic malignancy, ovarian cancer has the greatest mortality rate among them. Additionally cervical cancer is tremendously increasing especially in low socioeconomic regions. ${ }^{1}$ Although using surgery for primary treatment is still essential for especially gynecologic cancers, investigations are aimed for developing new treatment modalities for decreasing the mortality and morbidity due to malignancies.

Conventional chemotherapy regimens are highly effective at initial treatment process, during the treatment of the resistant cases and recurrences they loss their strength because of new mutation formation in the neoplastic tissue and other known/unknown processes of tumor cells. Recently new pathways for blocking tumor growth and dissemination are under research. Targeted therapy for tumor cells is one of these new approaches with direct and indirect methods. Despite chemotherapy affects rapidly dividing cells and may give suppression to gastrointestinal, myeloid system cells and hair growth, targeted therapies have a critical role on tumor growth, progression, invasion and metastasis by tumor cell selective therapy with less toxicity. Direct method can be briefly defined as altering tumor antigen signaling either by monoclonal antibodies (MoAbs) or by small molecule drugs on basis of target proteins. On the other hand, indirect method is against tumor antigens expressed on cell surface that is knotted by ligands. ${ }^{2}$

The most widely known targeted therapy option is monoclonal antibody treatment. They are monospecific antibodies which produced by one type of immune cell in laboratories and attach to specific areas in cancer cells. They recognize and bind to antigens and induce immune responses after binding. In mid 70's Kohler and Milstein describe a method for producing high amount of monoclonal antibodies named as Hybridoma Technology. ${ }^{3}$ First generation MoAbs were produced from murine, rabbit or rat proteins that purified after immunization with antigen which were highly allergic. Whereas second generation MoAbs which were produced with DNA technology show their antitumor effect by cell mediated cytotoxicity, complement dependent cytotoxicity, immunomodulation, altering signal transduction and immunoconjugates with less allergic reactions. The humanized molecular antibody, bevacizumab, which binds to circulating VEGF, is the most studied molecular therapeutic agent of these antiangiogenetic agents.

Plasma membrane associated protein tyrosine kinases are important enzymes for regular cell life. Mutations or over expressions have a role on tumor proliferation and angiogenesis. By the way small molecule inhibitors of protein kinases have a role on targeted therapy. ${ }^{4}$ The most important cytoplasmic signalling pathways are the phosphoinositide 3-kinase/AKT pathway, mammalian target of rapamycin (PI3K/AKT/mTOR), the Ras/Raf mitogen-activated protein kinase (MAPK) pathway, the Raf/MEK/Erk pathway and the protein kinase $\mathrm{C}$ pathway. ${ }^{5}$
Volume I Issue I - 2014

\author{
Emre Ozgu \\ Department of Gynecologic Oncology, Zekai Tahir Burak \\ Women's Health Research and Education Hospital,Turkey
}

Correspondence: Emre Ozgu, Department of Gynecologic Oncology, Zekai Tahir Burak Women's Health Research and Education Hospital, Ankara, Turkey, Tel +90-505-589-25-39, Email emreozgu@hotmail.com

Received: September 15, 2014 | Published: September 16, 2014

Tumor cells are in a high metabolic active state that presenting cytotoxic agents to tumor cells in a selective method is important for preventing harmful effects to other cells. In that manner ligand mediated targeted therapy is effective.

There are so many studies for defining specific target for the battle against tumor cells such as PI3K-AKT-mTOR pathway inhibitors, Hedgehog (Hh) Signalling, Aurora kinase inhibitors, Poly-ADPRibose Polymerase (PARP) inhibitors. All these investigations are aimed to define the most tumor cell specific response with minimum side effects to healthy cells. Solving the mysteries of the genomic genome helps us to understand the mechanism of cell cycle and gives us the opportunity to investigate more pathways for possible drug impact for tumor cells.

Cancer which is defined as plague of this era can no longer be a leading cause of mortality by the help of targeted therapy innovations. Future studies in oncology are seemed to be focused in this subject.

As a new journal, by the help of our colleagues' precious studies, we all hope to be a part of this innovative process.

\section{Acknowledgments}

None.

\section{Conflicts of interest}

Author has no any conflict of interest to declare.

\section{References}

1. Siegel R, Naishadham D, Jemal A. Cancer statistics. CA: A cancer Journal for Clinicians. 2013;63(1):11-30.

2. Wu HC, Chang DK, Huang CT. Targeted therapy for cancer. Journal of Cancer Molecules. 2006;2(2):57-66.

3. Kohler G, Milstein C. Continuous cultures of fused cells secreting antibody of predefined specificity. Nature. 1975;256(5517):495-497.

4. Knight ZA, Shokat KM. Features of selective kinase inhibitors. Chem Biol. 2005;12(6):621-637.

5. Manning G, Whyte DB, Martinez R, et al. The protein kinase complement of the human genome. Science. 2002;298(5600):1912-1934. 